\title{
ACTA TROPICA
}

\section{Use of thermal and vegetation index data from earth observing satellites to evaluate the risk of schistosomiasis in Bahia, Brazil}

\author{
M.E. Bavia ${ }^{\mathrm{a}, *}$, J.B. Malone ${ }^{\mathrm{b}}$, L. Hale ${ }^{\mathrm{c}}$, A. Dantas ${ }^{\mathrm{d}}$, L. Marroni ${ }^{\mathrm{e}}$, R. Reis ${ }^{\mathrm{a}}$ \\ ${ }^{a}$ Faculty of Veterinary Medicine, Universidade Federal da Bahia, Salvador, Brazil \\ ${ }^{\mathrm{b}}$ School of Veterinary Medicine, Louisiana State University, Baton Rouge, LA, USA \\ ${ }^{\mathrm{c}}$ Louisiana Department of Natural Resources, Baton Rouge, LA, USA \\ d Fundação Nacional de Saude, Salvador, Brazil \\ e Instituto de Pesquisas Espaciais, Cocheira Paulista, São Paulo, Brazil
}

\begin{abstract}
A geographic information system (GIS) was constructed using maps of regional agroclimatic features, vegetation indices and earth surface temperature data from environmental satellites, together with Schistosoma mansoni prevalence records from 270 municipalities including snail host distributions in Bahia, Brazil to study the spatial and temporal dynamics of infection and to identify environmental factors that influence the distribution of schistosomiasis. In an initial analysis, population density and duration (months) of the annual dry period were shown to be important determinants of disease. In cooperation with the National Institute of Spatial Research in Brazil (INPE), day and night imagery data covering the state of Bahia were selected at approximately bimonthly intervals in 1994 (six day-night pairs) from the data archives of the advanced very high resolution radiometer (AVHRR) sensor of the National Oceanic and Atmospheric Administration (NOAA)-11 satellite. A composite mosaic of these images was created to produce maps of: (1) average values between 0 and +1 of the normalized difference vegetation index (NDVI); and (2) average diurnal temperature differences $(\mathrm{d} T)$ on a scale of values between 0 and $15^{\circ} \mathrm{C}$. For each municipality, NDVI and $\mathrm{d} T$ were calculated for a $3 \times 3$ pixel $\left(9 \mathrm{~km}^{2}\right.$ area $)$ grid and analyzed for relationships to prevalence of schistosomiasis. Results showed a statistically significant relationship of prevalence to $\mathrm{d} T(\rho=-0.218)$ and NDVI $(\rho=0.384)$ at the $95 \%$ level of confidence by the Spearman rank correlation coefficient. Results support use of NDVI, dT, dry period climatic stress factors and human population density for development of a GIS environmental risk assessment model for schistosomiasis in Brazil. (C) 2001 Elsevier Science B.V. All rights reserved.
\end{abstract}

Keywords: Schistosomiasis; Schistosoma mansoni Epidemiology; Remote-sensing; GIS

\section{Introduction}

* Corresponding author. Tel.: + 55-71-7639868; fax: + 55-

Schistosomiasis is a complex water-borne dis71-2438189.

E-mail address: m_bavia@hotmail.com (M.E. Bavia). ease of medical, social, behavioral and ecological significance, considered second only to malaria in 
socio-economic and public health importance in tropical and sub-tropical areas (World Health Organization, 1993). Intestinal schistosomiasis was first reported in Brazil in 1902 by Piraja da Silva (Doumenge et al., 1987) in the northeast of the country. Since 1920, the endemic area has spread to other regions. Schistosomiasis in Brazil is due to S. mansoni and either Biomphalaria straminea, Biomphalaria glabrata or Biomphalaria tenagophila (Doumenge et al., 1987)

The geographical distribution of schistosomiasis is a function of the interaction between abiotic and biotic environmental factors. The disease has a focal distribution and requires contact with water containing infected snails. As with other zoonoses, schistosomiasis has a natural habitat in a well-defined ecosystem. Pathogens, vectors and natural hosts form an association or biocoenose within which the pathogen reproduces and is disseminated (Appleton, 1978). Temperature, rainfall, relative humidity and wind are known to have an important influence on a wide range of health processes, and all are predicted to be affected by future climate change (Beck et al., 2000). From previous work in 30 randomly selected municipalities, it was established that, in Bahia, regional differences in the prevalence of $S$. mansoni are determined more by the limiting effects of drought stress on life cycle development and transmission than by rainfall or temperature (Bavia, 1996).

Since the disease is constrained in space and time by environmental factors, geographic information systems (GIS) is useful in understanding the distribution of the parasites and their hosts in relation to maps depicting spatial and temporal features of the environment. The use of GIS and remote sensing (RS) to identify environmental features allows the determination of risk factors and delimitation of areas at risk, permitting more rational allocation of resources for cost-effective control (Beck et al., 1997; Beck et al., 2000). The application of products derived from the advanced very high resolution radiometer (AVHRR) sensor on board the National Oceanic and Atmospheric Administration (NOAA) series of environmental satellites has not previously been used to study schistosomiasis in Brazil. Earlier reports suggest that the Normalized Difference Vegetation Index (NDVI) and diurnal temperature difference values $(\mathrm{d} T)$ may be useful as surrogate measures of environmental moisture and thermal regimes for development of environmental risk models (Malone et al., 1997).

In Brazil, the traditional control program has included mass chemotherapy with supplemental use of snail control, a general practice that has been applied to endemic communities for more than 50 years. This basic approach has been successful in preventing mortality and reducing morbidity, but has not contained the expansion of the endemic area in the state of Bahia or elsewhere.

The objectives of this study were: (1) to determine the relationship between the distribution and abundance of schistosomisis in the state of Bahia, Brazil, and the suitability of the environment as measured by NDVI, diurnal temperature difference $(\mathrm{d} T)$, and length of the annual dry period; and (2) to use these factors to develop a multivariate model for the prediction of the environmental risk of the disease in the 230 endemic municipalities in Bahia, Brazil. The study proposes to include NDVI and $\mathrm{d} T$ as surrogate climatic indicators of regional surface hydrological regime that can be used to establish descriptive parameters relating to the spatial and temporal dynamics of schistosomiasis.

\section{Material and methods}

The state of Bahia, with an area of approximately $584000 \mathrm{~km}^{2}$ and $932 \mathrm{~km}$ Atlantic Ocean coastline, is divided politically into 417 municipalities where approximately 13 million people live under tropical climatic conditions (Fundacao Instituto Brasileiro de Geografia e Estatistica, 1995). Prevalence data from the National Foundation of Health (NFH) surveillance program for 19921994 were used as prevalence rates for the 230 municipalities included in the study. Prevalence rates were classified into five categories $(0-5,>5$ to $15,>15$ to $25,>25$ to 40 , and $>40$ to $90.4 \%)$. A map depicting the average length of the annual dry period (Nimer, 1989) was digitized at a scale of 1:1 million, converted to an ARCVIEW 
shapefile and represented as contours with from zero to 11 months of dryness. Population density was calculated according to 1994 data from the Brasilian Institute of Geography and Statistics (Fundacao Instituto Brasileiro de Geografia e Estatistica, 1995). Statistical analysis was carried out using stepwise multiple regression analysis and the Spearman rank correlation coefficient (Slott, 1990).

SPRING modular GIS software from the National Institute of Spatial Research (INPE), Sao Paulo, Brazil, was used to develop and analyze satellite-derived maps of NDVI and $\mathrm{d} T$ for these studies. A total of 230 endemic municipalities (of 417 total) were georeferenced on composite maps created from a 1994 time-series of six day-night pairs of AVHRR imagery acquired from INPE. Day-night pairs for 6 days and 6 nights at approximately $06: 00$ and $1500 \mathrm{~h}$ on 16 January, 5 March, 17 May, 20 July, 21 September and 17 November were calibrated, georeferenced and co-registered. Daytime Channel 1 visible and Channel 2 near-infrared data were used to calculate a NDVI average for each municipality using the formula: $\mathrm{Ch} 1-\mathrm{Ch} 2 / \mathrm{Ch} 1+\mathrm{Ch} 2=\mathrm{NDVI}$. A grid of $3 \times 3 \mathrm{~km}^{2}$ areas $(3 \times 3$ pixels $)$ was used to calculate the NDVI average value within the boundary of each municipality (Fig. 2a). Values for $\mathrm{d} T$ were measured by calculating the difference between daytime and night-time temperatures extracted from Channel 4 (thermal infrared) of the AVHRR sensor. The $\mathrm{d} T$ value was calculated from a grid of $3 \times 3 \mathrm{~km}^{2}$ areas by subtracting Channel 4 night-time from Channel 4 daytime data $(\mathrm{d} T=\mathrm{A}-\mathrm{B})$. This resulted in 19 $\mathrm{d} T$ values that were classified and represented as a $\mathrm{d} T$ map with eight $\mathrm{d} T$ classes ranging, in $2^{\circ}$ increments, from $0-1$ to $14-15$ (Fig. 2b).

\section{Results}

The variation in percent prevalence among communities in the NFH control program survey carried out between 1992 and 1994 ranged from 0 to $90.4 \%$. A prevalence rate of $0-5 \%$ was found in $57(24.8 \%)$ of the municipalities; of these, 54 showed prevalence rate values less than
$1 \%$ and only three of these communities were diagnosed as free of $S$. mansoni. In 66 municipalities $(28.7 \%)$, prevalence was $5-15 \%$. A prevalence of $15-25$ or $25-40 \%$ was found in 35 $(13.0 \%)$ municipalities each. Very high values (40-90.4\%) were found among individuals in 12 $(5.2 \%)$ of the municipalities. Reports of the NFH schistosomiasis campaign program record the presence of both $B$. glabrata and $B$. straminea in each of the 230 municipalities selected for this study. The presence of both snail species were validated as part of current studies in a 10\% random sample of villages in 19981999.

The average length of the dry season during a 30 -year period for the state of Bahia varies from 0 to 11 months of dryness (Nimer, 1989). The highest occurrence of high prevalence was reported in coastal areas with high population density and in areas where the length of dry period did not exceed 6 months of drought (Fig. $1)$.

Values of NDVI extracted from $3 \times 3 \mathrm{~km}$ areas from the annual composite of six daytime AVHRR sensor images were calculated and ranked within the value range of 0 to +1 (Fig. 2a). Diurnal temperature difference $(\mathrm{d} T)$ values were ranked from 0 to $15^{\circ} \mathrm{C}$ after similar extraction of data from an annual composite map prepared from six pairs of day/night images (Fig. 2b).

Spearman correlation analysis revealed that prevalence rates were inversely related to the length of the dry period and $\mathrm{d} T$, and directly related to NDVI and population density. Municipalities with high prevalence rates thus had high values for NDVI and low values for $\mathrm{d} T$. A stepwise multiple regression model developed using the $\log$ of prevalence as the dependent variable showed that the best two-variable model included dryness and NDVI values $(\rho=0.36 ; P<$ $0.0001 ; F=63.29$ ). A three-variable model that included population density (range, $19-50 / \mathrm{km}^{2}$ ), resulted in improved model values $(\rho=0.36$; $P<0.0001 ; F=43.41)$. The model was further improved using a four-variable model that included d $T$ values $(\rho=0.40 ; P<0.01 ; F=33.27)$. 


\section{Discussion}

In previous work (Bavia et al., 1999) on a sample of 30 municipalities in the state of Bahia that focused on hydrological features and temperature patterns, the length of the dry period and population density (range, 19-50 people $/ \mathrm{km}^{2}$, were shown to be the most important limiting factors on schistosomiasis distribution and abundance. The present study, which was expanded to include all 230 endemic municipalities of Bahia, was designed to validate these findings and to further investigate the relationship of regional moisture-thermal regime and risk of schistosomiasis using NDVI and $\mathrm{d} T$ values as climate surrogate data. Results of the present study: (1) validated the direct relationship to population density and indirect relationship to the length of the drought period in months as a major limiting stress factor on the development and transmission of the S. mansoni life cycle; and (2) indicated that satellite-derived vegetation index (NDVI) and diurnal temperature difference $(\mathrm{d} T)$ values from the AVHRR sensor can be used to define regional

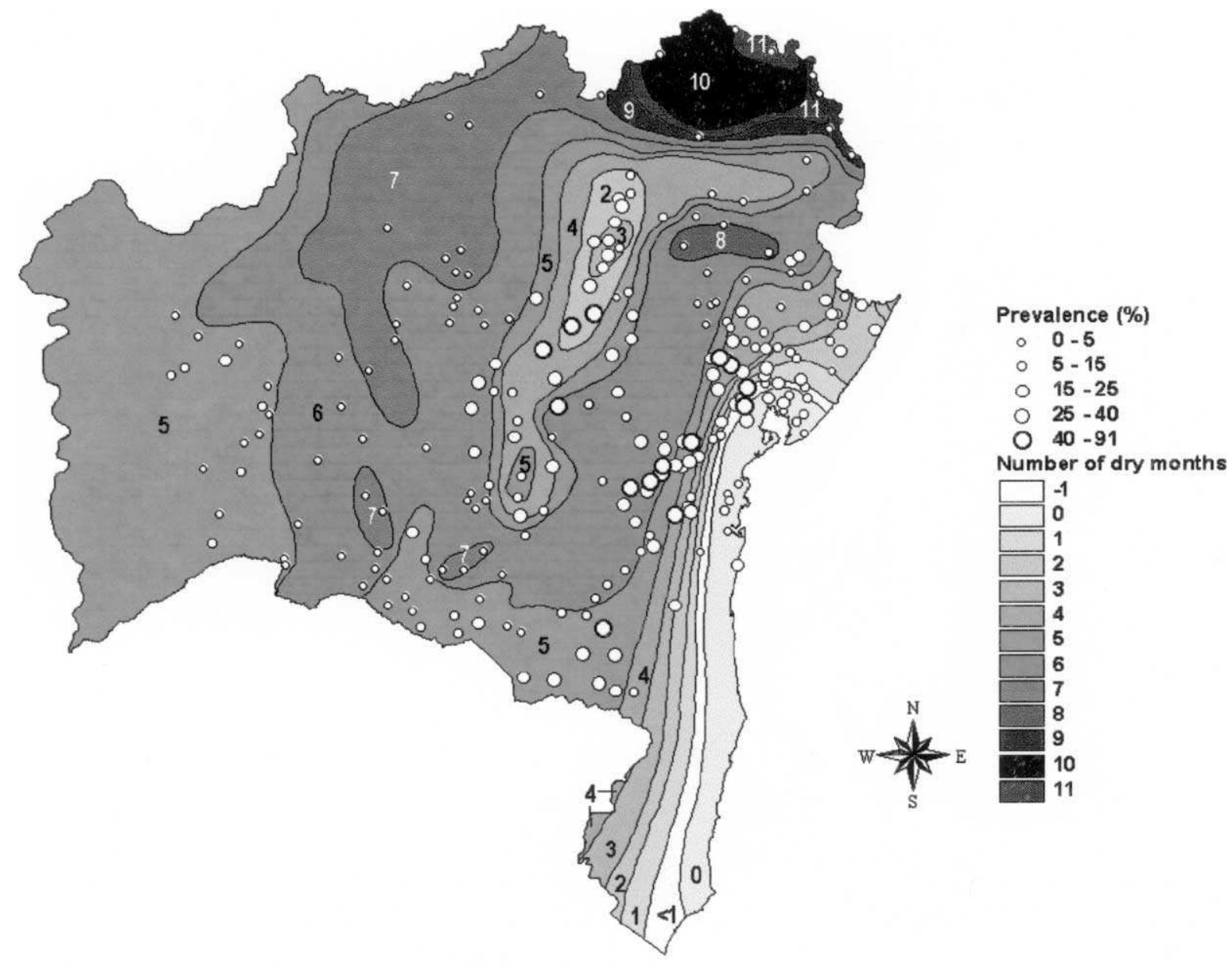

300 Kilometers

Fig. 1. Prevalence of S. mansoni in 230 endemic municipalities (of 431 total) in relation to the annual number of dry months. Note that municipalities with high to very high prevalence rates $(>25 \%)$ are clustered in areas with a $3-4$ month dry period. 

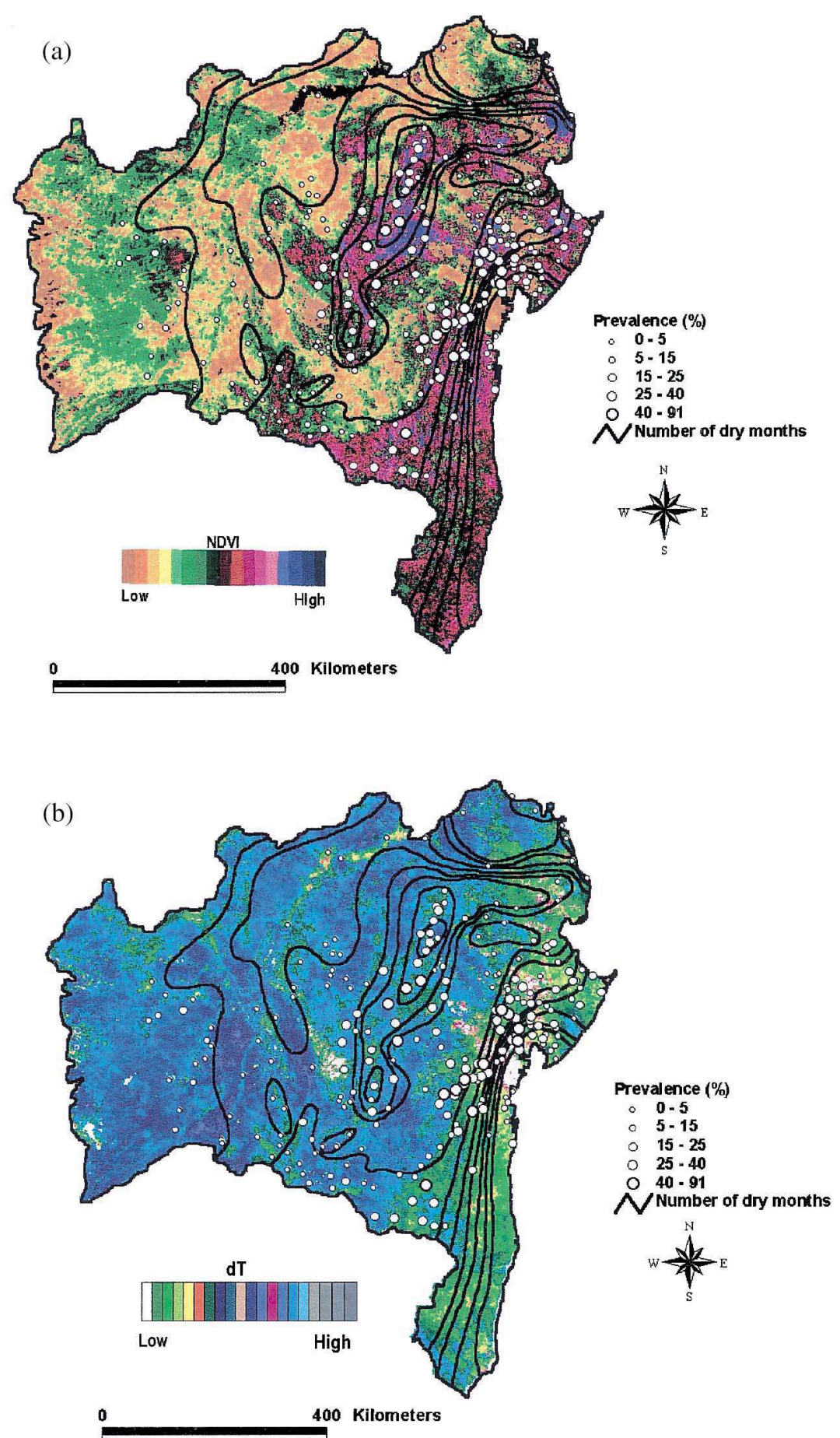

Fig. 2. Prevalence of $S$. mansoni and annual number of dry months overlaid on an annual composite NDVI map (a) (low $=0$ and high $=0.5)$ and a diurnal temperature difference $(\mathrm{d} T)$ annual composite map (b) (low $=0$ and high $=15)$ prepared from six day-night pairs of images (approximately bi-monthly intervals) from the AVHRR sensor of the NOAA series of earth observing satellites. Both NDVI and $\mathrm{d} T$ are proposed to be a surrogate measures of environmental thermal-hydrologic regime. The wettest sites have the lowest values of $\mathrm{d} T$ due to the buffering effect of environmental moisture on diurnal temperature fluctuation, and also have the highest value of NDVI due to the cumulative effects of increasing rainfall on vegetation growth and vigor. 
patterns of moisture-thermal regimes that favor the propagation and transmission of $S$. mansoni in Bahia, Brazil.

There was an inverse association between prevalence rates and the length of the dry period, and most communities with high schistosomiasis morbidity rates occur in areas that do not exceed 5 months of drought. Communities with high to very high prevalence rates $(25-40$ and $40-90.4 \%)$ are clustered in areas with a 3-4 month dry period (Fig. 1). However, Spearman correlation analysis showed that only $36 \%$ of the variation in schistosomiasis morbidity in the state of Bahia can be explained by regional variation in the length of dry period $(P<0.0001)$, and additional environmental risk parameters are needed to refine the ability to predict regional risk of schistosomiasis using GIS.

Remote sensing and GIS methods have been used to define and map landscape and moisture patterns that favor vector-borne disease habitats and to develop models, mainly at a regional scale, that define suitable conditions for development of the life cycles of snail host-parasite systems (Abdel-Rahman et al., 1997; Malone et al., 1997; Yilma and Malone, 1998). In the present investigation in Brazil, six day-night pairs of images from the AVHRR sensor of the NOAA-11 environmental satellite were acquired at approximately bi-monthly intervals to evaluate the strength of the association between schistosomiasis prevalence in Bahia and environmental moisture-thermal regime as measured by NDVI and $\mathrm{d} T$ values.

Diurnal temperature difference average values $(\mathrm{d} T)$ calculated from a grid of $3 \times 3 \mathrm{~km}^{2}(3 \times 3$ pixels) for each municipality were shown to have an inverse association with schistosomiasis prevalence (Fig. 2b). Although high-risk communities were associated with low values of temperature difference, $\mathrm{d} T$ as a single variable showed a relatively weak inverse relationship $(\rho=1.86, P<$ $0.0001)$ to the percentage of schistosomiasis in communities with above $25 \%$ prevalence rates. Normalized difference vegetation index average values, similarly obtained from a $3 \times 3 \mathrm{~km}^{2}$ area grid, had a direct relationship with schistosomiasis and can explain, by itself, $25 \%$ of the preva- lence variation within communities ( $\rho=0.25$ at the $P<0.0001$ confidence interval). The distribution sites with over $25 \%$ prevalence corresponded to relatively high values of NDVI, indicating the presence of adequate moisture at developmental temperatures favorable to $S$. mansoni. Overlays of the length of the annual dry period on annual composites maps prepared from 1994 NDVI and $\mathrm{d} T$ data demonstrate a clear relationship between these factors and percent prevalence rates (Fig. $2 \mathrm{a}, \mathrm{b})$, and suggest that each of these data sources may be useful components of more comprehensive GIS regional risk models for the management of schistosomiasis control programs in Brazil.

The intermediate snail hosts of schistosomiasis in Brazil are adapted to a wide range of environmental conditions. In Bahia, the amplitude of ambient temperature variation is around $5^{\circ} \mathrm{C}$. The rainfall pattern within the state is typically tropical (300-2000 mm/year) with a high concentration of rain during a few months, except in coastal areas where more extended precipitation occurs (McNally, 1990). Earlier reports suggest that temperature and rainfall regimes may be favorable to reproduction of the snail host and parasite in the entire state of Bahia in the future context of projected water resources development, human population increase and movements, persistent poverty and lagging control programs (Bavia et al., 1999). In 1996 and 1997, WHO reported schistosomiasis prevalence rates of $10.3 \%$ and $8.8 \%$ respectively, in the state of Bahia. Of the 230 communities examined, considered to be endemic by the National Foundation for Health, only three were found to be free of schistosomiases, although two of the intermediate hosts, $B$. straminea and B. glabrata, were observed there. In $33.4 \%$ of the communities, morbidity rates ranged from 0 to $5 \%$, suggesting that conditions currently exist for future expansion of the disease.

With the objective of preparing a model based on moisture-thermal regime risk that can be extrapolated to other areas with similar characteristics, a three-step multivariate stepwise regression model was developed that included values of population density, NDVI, and length of the annual dry period $(\rho=0.37 ; P<0.001 ; F=43.4)$. Inclusion of $\mathrm{d} T$ as a fourth variable to improve the 
accuracy of the three-step model resulted in an improved multivariate four-step regression model $(\rho=0.40 ; P<0.001 ; F=33.27)$. We propose the best current model for predicting regional variation in risk of schistosomiasis in the state of Bahia is represented by the four-variable model that includes NDVI, $\mathrm{d} T$, the length of the dry period and population density.

Patterns of moisture can be efficiently mapped over large areas using RS data, which provide an integrated view that is often not easily perceived from the ground and that enable predictions of risk in unsurveyed sites based on identification of moisture domains favorable to schistosomiasis. Although measurements of thermal-moisture regime provide broad limits to schistosomiasis distribution at regional scales, the well-known focality of the disease may be explained by a number of additional environmental requirements at local scales that must also be met to support the chain of reproduction of parasites, snails and final hosts. There is a need for a GIS environmental risk model that can provide accurate prediction for both regional and local scale risk areas in a single system. In response to this need, we are currently focusing on landscape features (hypsography, land use, soil type, slope, hydrology) using maps prepared from Landsat TM images and GPS points at five municipalities that are representative of environments in the 230 endemic municipalities. The goal is to test the predictive capability of the current regional four-step risk model in the state of Bahia by extrapolated to other endemic areas and to improve on the utility of the model at local scales i.e. $<1: 50000$ by modeling landscape features that can explain local variance in schistosomiasis prevalence rates.

\section{Acknowledgements}

The authors are grateful to the Life Science Division of the National Aeronautics and Space administration (NASA) via National Institutes of Health Grant 2 P50 AI 30639 (Core C) for financial support. They also thank the National Foun- dation of Health for providing field data and Bernardo Rudorf from INPE for remote sensing technical support. Deborah Duarte, Daniela Laranjeiras and Jennifer McCarroll provided indispensable technical help.

\section{References}

Abdel-Rahman, M.S., El-Bahy, M.M., El-Bahy, N.M., Malone, J.B., 1997. Development and validation of a satellite based geographic information system (GIS) model for epidemiology of Schistosoma risk assessment at snail level in Kafr El-Sheikh Governorate. J. Egypt. Soc. Parasitol. 27, 299-316

Appleton, C.C., 1978. Review of literature on abiotic factors influencing the distribution and life cycle of bilharziasis intermediate host snails. Malacol. Rev. 11, 1-15.

Bavia, M.E., Hale, L.F., Malone, J.B., Braud, D.H., Shane, S.M., 1999. Geographic information systems and the environmental risk of schistosomiasis in Bahia, Brazil. Am. J. Trop. Med. Hyg. 60, 566-572.

Bavia, M.E., 1996. Geographic information systems for schistosomiasis in Brazil. Ph.D. Dissertation. Louisiana State University, 199 pp.

Beck, L.R., Rodrigues, M.H., Dister, S.W., Rodriguez, A.D., Washino, R.T., Roberts, D.R., Spanner, M.A., 1997. Assessment of a remote sensing-based model for predicting malaria transmission risk in villages of Chiapas, Mexico. Am. J. Trop. Med. Hyg. 56, 99-106.

Beck, L.R., Lobitz, B.M., Wood, B.L., 2000. Remote sensing and human health: new sensors and new opportunities. Emerg. Inf. Dis. 6, 217-227.

Doumenge, J.P., Mott, K.E., Cheung, C., Villenave, D., Capui, O., Perrin, M.F., 1987. Atlas of the Global Distribution of Schistosomiasis. Talence, Geneva.

Fundacao Instituto Brasileiro de Geografia e Estatistica, 1995. Anuario Estatistico do Brasil. FIBGE, Rio de Janeiro.

Malone, J.B., Abdel-Rahman, M.S., El-Bahy, M.M., Huh, O.K., Shafik, M., Bavia, M.E., 1997. Geographic information systems and the distribution of Schistosoma mansoni in the Nile Delta. Parasitol. Today 13, 112-119.

McNally, R., 1990. Goode's World Atlas, 18th ed. E.B. Espenshad, New York.

Nimer, E., 1989. Climatologia do Brasil. FIBGE, Rio de Janeiro.

Slott, S., 1990. Statistics for Health Professionals. W.B. Saunders, Philadelphia, PA.

World Health Organization, 1993. The control of schistosomiasis. Second Report of the WHO Expert Committee, WHO Technical Report Series 830. WHO, Geneva.

Yilma, J.M., Malone, J.B., 1998. A Geographic information system forecast model for strategic control of fasciolosis in Ethiopia. Vet. Parasitol. 78, 103-127. 